Article

\title{
One-Step Hydrothermal Synthesis of Yellow and Green Emitting Silicon Quantum Dots with Synergistic Effect
}

\author{
Zhixia Zhang ${ }^{1,2}$, Chunjin Wei ${ }^{1,2}$, Wenting Ma ${ }^{1,2}$, Jun Li ${ }^{1,2}$, Xincai Xiao ${ }^{1,2}$ \\ and Dan Zhao 1,2,*(D) \\ 1 School of Pharmaceutical Sciences, South-Central University for Nationalities, Wuhan 430074, China; \\ zhixia_z@163.com (Z.Z.); wcj407704@163.com (C.W.); tingwm1993@163.com (W.M.); \\ lijun-pharm@hotmail.com (J.L.); xcxiao@126.com (X.X.) \\ 2 National Demonstration Center for Experimental Ethnopharmacology Education (South-Central University \\ for Nationalities), Wuhan 430065, China \\ * Correspondence: wqzhdpai@163.com; Tel.: +86-1806-208-4690
}

Received: 19 January 2019; Accepted: 11 March 2019; Published: 20 March 2019

check for updates

\begin{abstract}
The concept of synergistic effects has been widely applied in many scientific fields such as in biomedical science and material chemistry, and has further attracted interest in the fields of both synthesis and application of nanomaterials. In this paper, we report the synthesis of long-wavelength emitting silicon quantum dots based on a one-step hydrothermal route with catechol (CC) and sodium citrate (Na-citrate) as a reducing agent pair, and $\mathrm{N}$-[3-(trimethoxysilyl)propyl]ethylenediamine (DAMO) as silicon source. By controlling the reaction time, yellow-emitting silicon quantum dots and green-emitting silicon quantum dots were synthesized with quantum yields (QYs) of $29.4 \%$ and $38.3 \%$ respectively. The as-prepared silicon quantum dots were characterized by fluorescence (PL) spectrum, UV-visible spectrum, high resolution transmission electron microscope (HRTEM), Fourier transform infrared (FT-IR) spectrometry energy dispersive spectroscopy (EDS), and Zeta potential. With the aid of these methods, this paper further discussed how the optical performance and surface characteristics of the prepared quantum dots (QDs) influence the fluorescence mechanism. Meanwhile, the cell toxicity of the silicon quantum dots was tested by the 3-(4,5-dimethylthiazolyl-2)-2,5-diphenyltetrazolium (MTT) bromide method, and its potential as a fluorescence ink explored. The silicon quantum dots exhibit a red-shift phenomenon in their fluorescence peak due to the participation of the carbonyl group during the synthesis. The high-efficiency and stable photoluminescence of the long-wavelength emitting silicon quantum dots prepared through a synergistic effect is of great value in their future application as novel optical materials in bioimaging, LED, and materials detection.
\end{abstract}

Keywords: silicon quantum dots; synthesis; one-pot hydrothermal method; synergistic effect

\section{Introduction}

As a newcomer to nanomaterials, silicon quantum dots (SiQDs) have recently attracted tremendous attention. SiQDs exhibit tunable fluorescence emission properties, chemical stability, favorable biocompatibility, and low toxicity. Thus, they have shown promising potential in a wide range of fields, including lithium-ion batteries [1,2], biological imaging [3,4], and therapy $[5,6]$.

At present, most prepared SiQDs are blue-emitting ones $\left(\lambda_{\mathrm{em}}<450 \mathrm{~nm}\right)[7,8]$, but the short excitation wavelength $\left(\lambda_{\mathrm{ex}}\right)$ greatly limits their application in biochemical detection and imaging because of the background fluorescence interference. Therefore, the synthesis of long wavelength SiQDs has become a focus of researchers. Currently, methods for long wavelength emitting SiQDs 
synthesis are limited, among which the electrochemical method is commonly used. For example, Tu et al. [9] prepared red-emitting SiQDs by etching a p-type silicon wafer in an electrolyte containing HF and methanol. Kang et al. [10] synthesized SiQDs with an emission range from blue to red through a refluxing route after electrolyzing the silicon wafer in an electrolytic cell. Erogbogbo et al. [11] by pyrolyzing silane and etching, finally acquired red, yellow and green-emitting SiQDs. Besides, Kauzlarich et al. [12] acquired orange-emitting SiQDs by reflexing $\mathrm{Mg}_{2} \mathrm{~S}$ and adding normal-butyl blocking through a wet chemical method. However, these reported methods are quite complicated with obvious drawbacks such as expensive experimental equipment and high energy costs. Therefore, the development of new, simple, low energy cost, and environment-friendly synthesis methods have aroused immense interest. As a "bottom-to-up" synthesis method for SiQDs, the one-step hydrothermal route is simple in operation, and SiQDs prepared by this method have excellent dispersity without any requirement for further modification before their practical application. So far, most reported SiQDs synthesized though the one-step hydrothermal route emit blue light $[7,8,13]$, and only a few teams have synthesized green-emitting SiQDs by selecting proper reducing agents [14-16]. In a recent work, Ma et al. [14] obtained green-emitting SiQDs with (3-Aminopropyl)triethoxysilane (APTES) as silicon source and ascorbic acid as reducing reagent (QYs $=8.2 \%$ ). Wang et al. [15] acquired green-emitting SiQDs by choosing APTES as silicon source and sodium ascorbate as reducing reagent (QYs $=21 \%$ ). Han et al. [16], by using DAMO as silicon source and CC as reducing reagent, finally prepared green-emitting SiQDs with QYs at 7.1\%. However, the requirement for a large amount of raw materials, the low QYs, and the weak stability of prepared SiQDs turn out to be drawbacks of these syntheses. To overcome the disadvantages, synergistic effects have been introduced to improve the one-step hydrothermal route, to acquire SiQDs with better optical properties, chemical stability and high QYs.

The synergistic effect has been widely applied in the fields of material synthesis [17,18], pharmacology [19,20], and chemistry [21,22], as well as in chemical synthesis, and has been proven to effectively improve the output of the products and simplify the synthesis process. For example, Chen et al. [23] employed the synergistic effect between the metal-organic framework Pd@MOF and metal nanoparticles PdNPs to realize a one-step multiple cascade reaction to synthesize secondary arylamines; Haddleton [24] and his team used a congregation of photo-radicals based on the synergistic effect between $\mathrm{CuBr}_{2}$ and tertiary amine, to speed up the synthesis of acrylate and improve the monomer conversion rate, and acquired polyacrylate with excellent uniformity and stability. Moreover, the synergistic effect also shows its effectiveness in the synthesis of fluorescence nanomaterial. The synergistic effect between different elements during the synthesis of N/S [25] or N/P [26] co-doped carbon dots could enhance the optical-chemical activity of prepared carbon dots (CDs). Recently, our team [27] reported a synergistic effect synthesis strategy for preparing SiQDs with super-high QYs (84.92\%) by choosing Na-citrate and thiourea as reagent pair. Under optimal excitation environment, the prepared SiQDs emit blue fluorescence $\left(\lambda_{\mathrm{em}}=452 \mathrm{~nm}\right)$. Therefore, the impact of the synergistic effect of double reducing reagents upon the preparation of long-wavelength-emitting QDs was proven to be effective and positive.

In this study, we report a facile one-step route to prepare stable long-wavelength-emitting SiQDs via the synergistic effect between the reducing reagents $C \mathrm{C}$ and Na-citrate. The impacts of different synthesis environment parameters upon the optical properties of prepared SiQDs were explored. It was discovered that the reaction temperature could effectively adjust the emission wavelength $\left(\lambda_{\mathrm{em}}\right)$ of the product. Besides, we compared the SiQDs synthesized with the reagent pair (CC and Na-citrate) and SiQDs synthesized with only Na-citrate as reagent. The size, surface properties, and elemental composition of these two SiQDs were investigated to reveal their optical characteristics and the emission mechanism. The synergistic effect between the double reducing reagents was proven to be effective in the synthesis of stable long-wavelength-emitting SiQDs with efficient photoluminescence, and is of certain significance in the application as an ideal optical material. 


\section{Materials and Methods}

\subsection{Reagents and Instruments}

Sodium citrate $(99.0 \%)$ was obtained from Shanghai Zhan Yun Chemical Co., Ltd. (Shanghai, China). N-[3-(Trimethoxysilyl)propyl]ethylenediamine (95.0\%) and catechol ( $\geq 99.0 \%)$ were purchased from Aladdin Chemistry Co., Ltd., (Shanghai, China) Sodium sulfite ( $\geq 99.0 \%)$, ascorbic acid $(\geq 99.0 \%)$, thiourea $(\geq 99.0 \%)$, urea $(\geq 99.0 \%)$, dimethyl sulfoxide (DMSO) $(\geq 99.5 \%)$ and acetonitrile $(\geq 99.0 \%)$ were obtained from Sinopharm Chemical Reagent Co., Ltd. (Shanghai, China). MTT ( $\geq 98.0 \%)$ was purchansed form Sigma-Aldrich Co., Ltd. (Merck KGaA, Darmstadt, Germany). All solutions were prepared using Milli-Q water (Millipore, Burlington, MA, USA) as the solvent.

UV-visible absorption spectra were acquired with a Lambda-35 UV-visible spectrophotometer (PerkinElmer Company, Waltham, MA, USA) to determine the bandgap absorption of SiQDs. Fluorescence spectra were recorded on a LS55 spectrofluorometer (PerkinElmer Company). HRTEM images were obtained with a JEM2100F transmission electron microscope (Japan Electron Optics Laboratory Company, Tokyo, Japan) and EDS data were obtained by its annex. FT-IR spectra were obtained on a Nicolet 6700 spectrometer (Thermo Fisher Scientific, Waltham, MA, USA). Zeta-potential measurement was carried out on a Zetasizer nanoseries ZEN3690 (Malvern, UK). The relative QYs of as-prepared SiQDs were measured according to the literature with Rhodamine $6 \mathrm{G}$ in ethanol (QY $=95 \%)$ as a reference standard. All optical measurements were performed at room temperature under ambient conditions.

\subsection{Preparation of Silicon QDs}

Na-citrate $\left(9.24 \times 10^{-5} \mathrm{~mol} \cdot \mathrm{L}^{-1}\right)$ was dissolved in water $(10 \mathrm{~mL})$ under stirring for $10 \mathrm{~min}$. Then, DAMO $(0.2 \mathrm{~mL})$ was added to the solution and stirring continued for $10 \mathrm{~min}$. Finally, $1.1 \mathrm{mg}$ CC was added to the solution with stirring for $1 \mathrm{~min}$. Subsequently, the resulting products were heated in a Teflon-equipped stainless-steel autoclave at $130{ }^{\circ} \mathrm{C}$ or $150{ }^{\circ} \mathrm{C}$ for $5 \mathrm{~h}$. After naturally cooling to room temperature, four volumes of acetonitrile were added to the obtained solution and the mixture was centrifuged at $8000 \mathrm{rpm}$ for $15 \mathrm{~min}$ to remove raw material.

The same concentration of sodium sulfite, ascorbic acid, thiourea, or urea was used instead of $\mathrm{Na}$-citrate, and heated at $130{ }^{\circ} \mathrm{C}$ for $5 \mathrm{~h}$ to obtain SiQDs of different reducing agent combinations to compare their relative quantum yields. The relative PLQYs of as-prepared SiQDs were measured according to the literature with Rhodamine 6G in ethanol (QY $=95 \%)$ as a reference standard.

\subsection{MTT Method}

The MTT method was used to detect the cell viability of two kinds of SiQDs. The L02 cells were seeded at a density of $1 \times 10^{4}$ cells per well with $100 \mu \mathrm{L}$ of culture medium in 96-well plates and placed for cell growth for $24 \mathrm{~h}$ in a $37^{\circ} \mathrm{C}, 5 \% \mathrm{CO}_{2}$ humidified incubator. The appropriate amount of yellow-emitting SiQDs (y-SiQDs) and green-emitting SiQDs (g-SiQDs) were dissolved in ultrapure water and added to Dulbecco's modified eagle medium (DMEM) to a concentration of $200 \mu \mathrm{g} / \mathrm{mL}$. Different concentrations of dilution samples were added per well (The concentration of DMSO was less than 1\%o). After adding $100 \mu \mathrm{L}$ of MTT solution to each well, it was placed in the incubator for $30 \mathrm{~min}$. Its supernatant was discarded after culturing the cells for $24 \mathrm{~h}$, and then $150 \mu \mathrm{L}$ DMSO per well was added. After shaking in the dark for $10 \mathrm{~min}$, the microplate reader detected the optical density value (OD) at $562 \mathrm{~nm}$. The cell survival rate is calculated as follows. IC50 values were calculated by GraphPad Prism 6 (GraphPad Software, Inc., San Diego, CA, USA).

$$
\text { Cell viability }(\%)=\frac{\text { OD value of experiment group }- \text { OD value of control group }}{\text { OD value of negative control group }- \text { OD value of control group }}
$$




\section{Results and Discussion}

\subsection{The Optimization of the Synthesis Environment for SiQDs}

\subsubsection{Filtration of the Proper Selection of Reducing Reagents for SiQDs Preparation}

The reducing reagent is one of the most important factors of the synthesis that determines the optical properties of the prepared SiQDs. Proper selection of reducing reagents can effectively change the fluorescence intensity and $\lambda_{\mathrm{em}}$ of the products. Based on the published literature and our previous work, it was discovered that ascorbic acid (VitC) [14], sodium ascorbate (VitC-Na) [15], and CC [16,27] are the ideal candidates for the preparation of long-wavelength emitting SiQDs. The preparation process with one of them as reducing reagent could produce green-emitting SiQDs $\left(\lambda_{\mathrm{em}} \sim 520 \mathrm{~nm}\right)$, but the QYs of the production were quite low $(<5 \%)$. Adjusting the traditional synthesis environment, such as the adjustment of reactant ratio, reaction time, and temperature, could not further improve the optical properties of the product. Therefore, this paper reports the introduction of a second reducing reagent into the synthesis process, to improve the stability and QYs of the products through a synergistic effect.

Since the synergistic effect between double reducing reagents has been proven to be effective in improving the optical properties of blue-emitting SiQDs [27], the basic reducing reagent should first be selected out of the above-mentioned three candidates (VitC, VitC-Na, and CC). Based on that, the second reducing reagent should be selected to combine with the basic reagent to improve the optical and chemical properties of the SiQDs. As is known, Na-citrate is an excellent reducing reagent [28]. The prepared SiQDs exhibit QYs as high as 73.3\% with DAMO as silicon source and Na-citrate as reducing reagent. Na-citrate was thus chosen as one candidate of the reducing reagent pair. Then, three other reducing reagent candidates were matched with Na-citrate to test their ability for improvement. With the reactant ratio fixed at n(DAMO):n(CC):n(Na-Citrate) = 1:0.11:0.47 $\left(\mathrm{cDAMO}=8.29 \times 10^{-2} \mathrm{~mol} \cdot \mathrm{L}^{-1}\right)$, reaction temperature at $130^{\circ} \mathrm{C}$, and reaction time for $5 \mathrm{~h}$, the prepared SiQDs emitted yellow fluorescence with Na-citrate/CC as reducing reagent pair. They also maintained excellent optical properties and chemical stability with QYs as high as $29.4 \%$. Na-citrate/VitC and Na-citrate/VitC-Na pairs could be used to prepare green-emitting SiQDs $\left(\lambda_{\mathrm{em}} \sim 500 \mathrm{~nm}\right)$ with QYs of $5.5 \%$ and $6.4 \%$. Comparing with the results of the three groups, CC was selected as one of the reducing reagents for the synergistic effect.

With CC chosen as one candidate, the other reducing reagent of the pair was changed to assess more pairing possibilities. In the synthesis process, the inorganic reducing reagent sodium sulfite, organic reducing reagents thiourea, urea, and $\mathrm{VitC}$ have all been proven to have excellent reducing abilities. As shown in Figure 1, the combinations of CC and any one of these reducing reagents also resulted in acquiring long-wavelength emitting SiQDs, with their QYs all higher than 15\%, obviously higher than that of the SiQDs prepared by the single reducing reagent CC. This proves that the synergistic effect of double reducing reagents could effectively improve the optical properties of SiQDs. This might be attributable to the strong reducing abilities of Na-citrate, as well as the unique structure of CC which is beneficial for the red-shift of $\lambda_{\mathrm{em}}$ of the products. More detailed discussion on the synthesis mechanism is presented in the later part of this paper. 


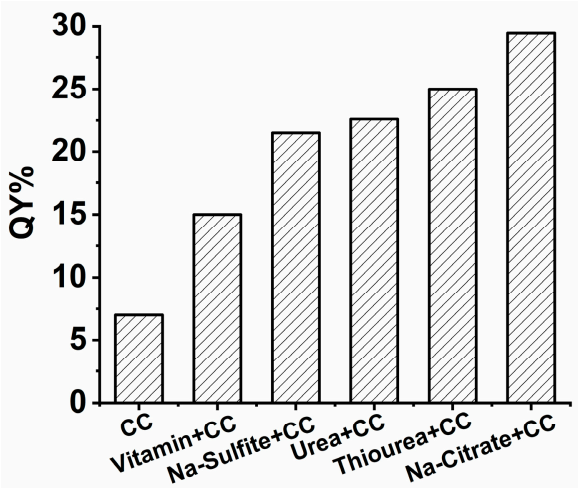

Figure 1. QYs of SiQDs synthesized with single reductant (CC) and double reductants (CC with other reductants).

\subsubsection{The Impact of Synthesis Parameters upon the Optical Properties of SiQDs}

The ratio of reactants, reaction temperature, and time are the key factors for SiQDs synthesis. Figure $2 \mathrm{a}$ shows the impact of the addition mass of Na-citrate. Fixing the reaction materials ratio of $\mathrm{c}(\mathrm{DAMO}): \mathrm{c}(\mathrm{CC})$ at 1:0.11, the solution was heated at $130{ }^{\circ} \mathrm{C}$ for $5 \mathrm{~h}$. When the addition mass of Na-citrate was raised to $13.6 \mathrm{mg}$, the fluorescence intensity of the prepared SiQDs reached a maximum. However, the changed amounts of Na-citrate did not change the $\lambda_{\mathrm{em}}$ of the prepared SiQDs. Changing the addition amount of CC, on the other hand, had an impact upon the $\lambda_{\mathrm{em}}$. As shown in Figure $2 b$, with other synthesis parameters fixed, and n(DAMO):n(Na-Citrate) set at 1:0.47, the $\lambda_{\mathrm{em}}$ of SiQDs redshifts from $518 \mathrm{~nm}$ to $546 \mathrm{~nm}$ with increasing CC amount. The QYs of the prepared SiQDs reach their maximum at $29.4 \%$ with the amount of CC rising to $1.1 \mathrm{mg}$.
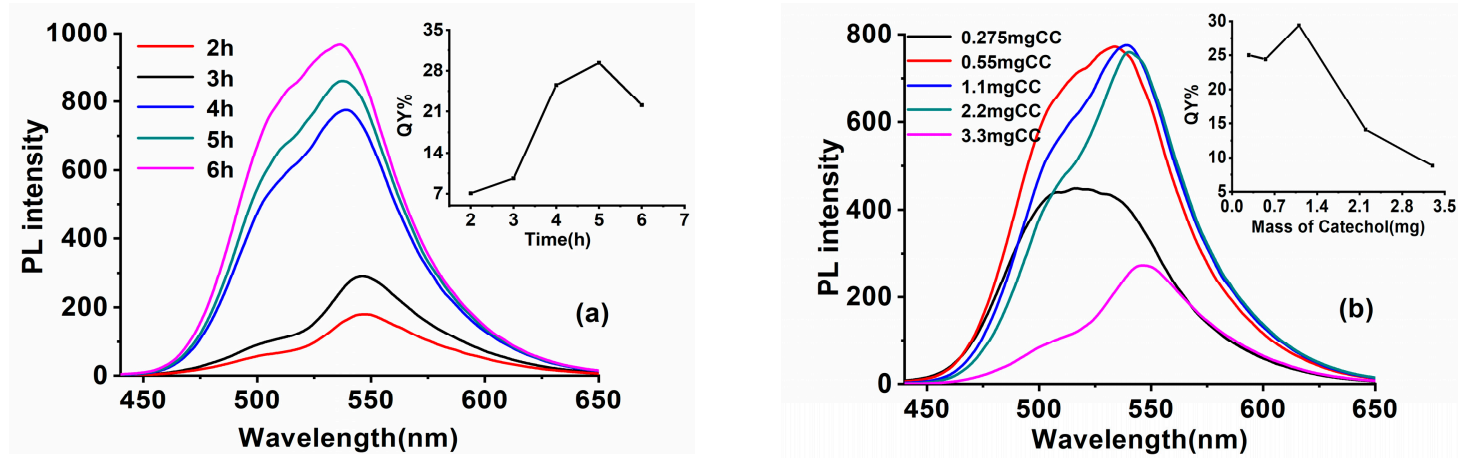

Figure 2. PL spectra of SiQDs synthesized with CC and Na-Citrate (a) with different addition mass of Na-citrate (b) with different addition masses of CC. The insets show the corresponding QYs.

On fixing the other synthesis parameters, the increased reaction time from $2 \mathrm{~h}$ to $6 \mathrm{~h}$ enhanced the fluorescence intensity of the prepared SiQDs, with the $\lambda_{\mathrm{em}}$ blue shifts from $546 \mathrm{~nm}$ to $535 \mathrm{~nm}$ (Figure 3a). The QYs of SiQDs reach their maximum with a reaction time of $5 \mathrm{~h}$.

Reaction temperature is also one of the important factors affecting the optical properties of SiQDs. Figure $3 \mathrm{~b}$ displays the different regular patterns of the QDs syntheses: the drop of reaction temperature from $150{ }^{\circ} \mathrm{C}$ to $110{ }^{\circ} \mathrm{C}$ renders $\lambda_{\mathrm{em}}$ red shifts from $520 \mathrm{~nm}$ to $545 \mathrm{~nm}$. A significant change in the color of SiQDs from green to orange could be observed under UV light. Surface chemistry is the key factor that determines the $\lambda_{\mathrm{em}}$ change of the prepared SiQDs [29]. The long-time exposure in the high-temperature oxidation environment would destroy the structure of $\mathrm{CC}$, preventing it from forming luminophores on the surface of the SiQDs while the silane mostly combines with Na-citrate, leading to short-wavelength emitting SiQDs. On the other hand, the low temperature environment would greatly decrease the fluorescence intensity. The QYs of prepared SiQDs dropped from 39.3\% 
to $9.4 \%$ when the reaction temperature dropped from $150{ }^{\circ} \mathrm{C}$ to $110{ }^{\circ} \mathrm{C}$. That may because the low temperature is not beneficial to the decomposition of silane to prevent the surface defects of the SiQDs.
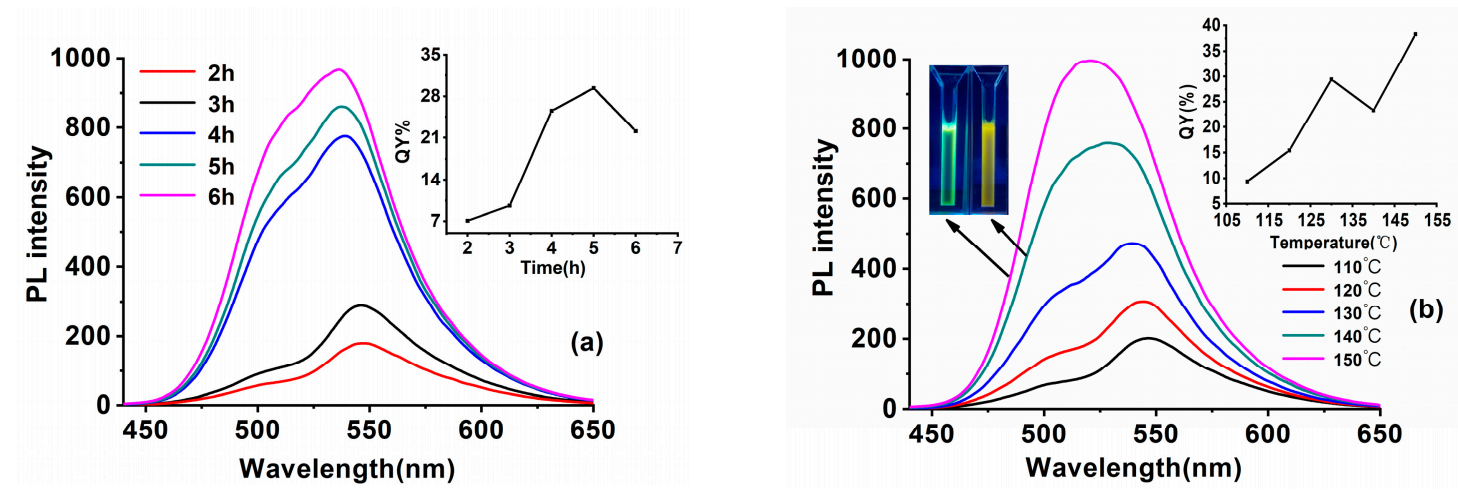

Figure 3. PL spectra of SiQDs synthesized with CC and Na-citrate at (a) different reaction time and (b) at different reaction temperature. Inset: the picture shows the increasing trend of QYs and the photos are of g-SiQDs and y-SiQDs.

Therefore, through a series of experiments, the best synthesis environment for high QYs yellow-emitting SiQDs (y-SiQDs) and green-emitting SiQDs (g-SiQDs) were acquired. As shown in the insert of Figure 3b, with n(DAMO):n(CC):n(Na-Citrate) fixed at 1:0.11:0.47, reaction time at $5 \mathrm{~h}$, y-SiQDs were acquired at a temperature of $130{ }^{\circ} \mathrm{C}$ with QYs at $29.4 \%$, and g-SiQDs were acquired at $150{ }^{\circ} \mathrm{C}$ with QYs at 38.3\%. Though both were prepared with the same raw materials, these two SiQDs exhibited different optical properties. The fluorescence emission peak of y-SiQDs (Figure S1) is obviously asymmetric, and two obvious UV absorption peaks can be observed at 233 and $254 \mathrm{~nm}$, while the g-SiQDs (Figure S2) does not exhibit a similar spectra shape.

\subsection{Characterization of SiQDs and Mechanism Discussion}

\subsubsection{Three Dimensional Fluorescence Spectra}

The three-dimensional fluorescence spectra could directly describe the changes of $\lambda_{\mathrm{em}}$ and fluorescence intensity with the $\lambda_{\mathrm{ex}}$. It was thus used to compare the optical property differences of the b-SiQDs (DAMO as silicon source, Na-citrate as reducing agent), SiQDs(CC) (DAMO as silicon source, CC as reducing agent) y-SiQDs, and g-SiQDs.

Figure $4 \mathrm{a}$ shows the change of the $b$-SiQDs fluorescence emission spectra with $\lambda_{\mathrm{ex}}$ in the range of 300-420 nm (gap at $10 \mathrm{~nm}$ ). With the increase of $\lambda_{\mathrm{ex}}$, the fluorescence intensity continues to increase and reaches a maximum at $\lambda_{\mathrm{ex}}=370 \mathrm{~nm}$. Further increase of $\lambda_{\mathrm{ex}}$ leads to a rapid decrease of fluorescence intensity, with its $\lambda_{\text {em }}$ staying at about $453 \mathrm{~nm}$.
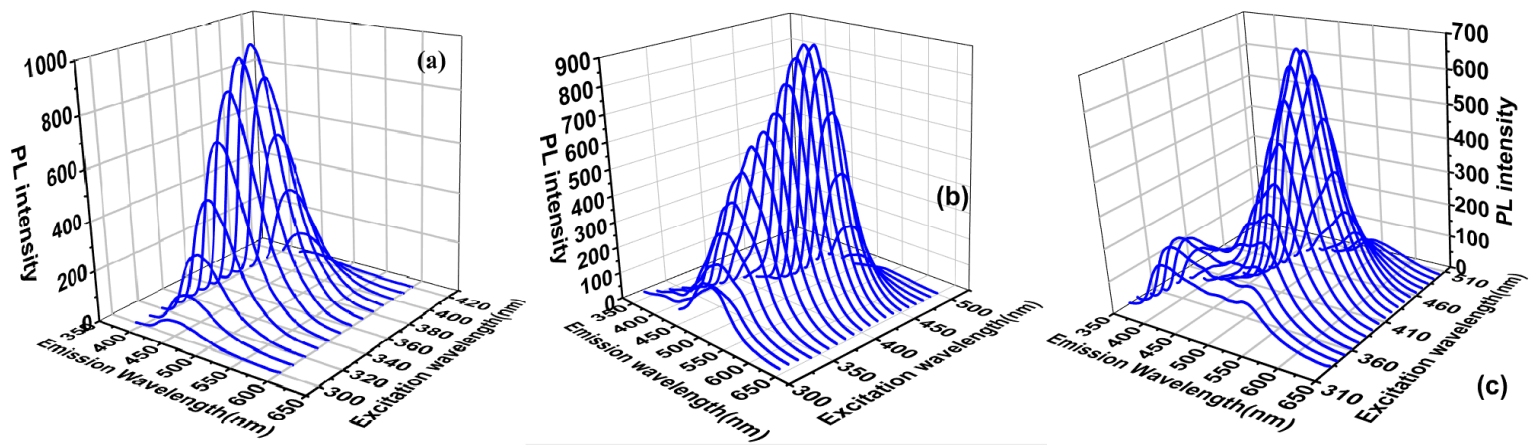

Figure 4. Three-dimensional fluorescence spectra of the three kinds of (a) b-SiQDs; (b) g-SiQDs, and (c) $y$-SiQDs. 
Figure S3 shows the three-dimensional fluorescence spectra comparing the three-dimensional spectra of SiQDs $(C C)$. We can find that, the $\lambda_{\mathrm{em}}$ of SiQDs(CC) only changes in fluorescence intensity at $550 \mathrm{~nm}$ with the $\lambda_{\text {ex }}$ of SiQDs(CC) increasing from $310 \mathrm{~nm}$ to $500 \mathrm{~nm}$. Also there is no occurrence of emission peak shift, which is similar to the cas of the three-dimensional spectra of b-SiQDs.

Figure $4 \mathrm{~b}$ exhibits a change of g-SiQDs fluorescence emission spectra with $\lambda_{\mathrm{ex}}$ in the range of 310-500 nm (gap at $10 \mathrm{~nm}$ ). The emission peak of g-SiQDs exhibits slight blue-shift when $\lambda_{\text {ex }}$ increases in the range of 310-360 nm, with gradual increase of fluorescence intensity. Further increase of $\lambda_{\mathrm{ex}}$ (360-420 nm) makes the emission peak redshift with further increase of fluorescence intensity, while the fluorescence intensity reaches the maximum $\left(\lambda_{\mathrm{em}}=527 \mathrm{~nm}\right)$ at $\lambda_{\mathrm{ex}}=420 \mathrm{~nm}$. When $\lambda_{\mathrm{ex}}$ reaches the range of 430-500 $\mathrm{nm}$, the redshifts of the emission peak continue with decrease of fluorescence intensity.

However, the change of $\mathrm{y}$-SiQDs in their three-dimensional fluorescence spectra is more complicated than the other two. Figure 4c displays the change of the $\mathrm{y}$-SiQDs fluorescence emission spectra with $\lambda_{\text {ex }}$ in the range of 330-550 nm (gap at $10 \mathrm{~nm}$ ), with two different changes in behavior being observed. When $\lambda_{\text {ex }}$ is in the range of 330-370 nm, two emission peaks exist at $395 \mathrm{~nm}$ and 540 $\mathrm{nm}$. With the increase of $\lambda_{\mathrm{ex}}$, the fluorescence intensity at $395 \mathrm{~nm}$ decreases, while the one at $540 \mathrm{~nm}$ enhances. When $\lambda_{\text {ex }}$ rises up to the range of 380 to $500 \mathrm{~nm}$, the emission peak at $395 \mathrm{~nm}$ disappears, and the fluorescence intensity of $y$-SiQDs increases with $\lambda_{\mathrm{em}}$. The intensity reaches a maximum at $\lambda_{\mathrm{ex}}=420 \mathrm{~nm}$. Further increase of $\lambda_{\mathrm{em}}$ would lead to redshift of the emission peak and decrease of fluorescence intensity.

Therefore, the particle size distribution results of $y$-SiQDs were used to demonstrate the possibility of the presence of two sizes of nanoparticles in y-SiQDs. As shown in Figure S4a, when performing the particle size distribution statistics of $\mathrm{y}$-SiQDs, the number of particles around $\sim 5 \mathrm{~nm}$ is also higher than the normal level, in addition to the large particles of $y$-SiQDs. This shows that the complex behavior of the 3D spectra of $y$-SiQDs is likely to originate from the presence of two types of SiQDs in the sample. This phenomenon may be due to a competitive reaction between the two reducing agents and the silicon source during the synthesis. Na-citrate is an excellent reducing agent that is more easily combined with silane to produce blue-emitting SiQDs.

\subsubsection{HRTEM Imaging}

The particle size of SiQDs is one of the crucial factors for its optical properties. Thus, the transmission electron microscope was applied to study the particle size and morphology of the prepared b-SiQDs and y-SiQDs. Both SiQDs exhibited uniform distribution and spherical morphology (Figure 5). The diameter distribution of the b-SiQDs is in the range of $2-3.5 \mathrm{~nm}$ with average diameter at $2.8 \mathrm{~nm}$; while the diameter distribution of $\mathrm{y}$-SiQDs is in the range of 10-13 $\mathrm{nm}$ with average diameter at $12 \mathrm{~nm}$. Figures S4 and S5 shows the particle size distribution histograms and the lattice plane of two kinds of SiQDs corresponds to the d-spacing of the cubic diamond structure of silicon giving the (111) plane with $0.31 \mathrm{~nm}$ spacing. It is thus proven that the increase of particle size could effectively make the emission peak redshift.
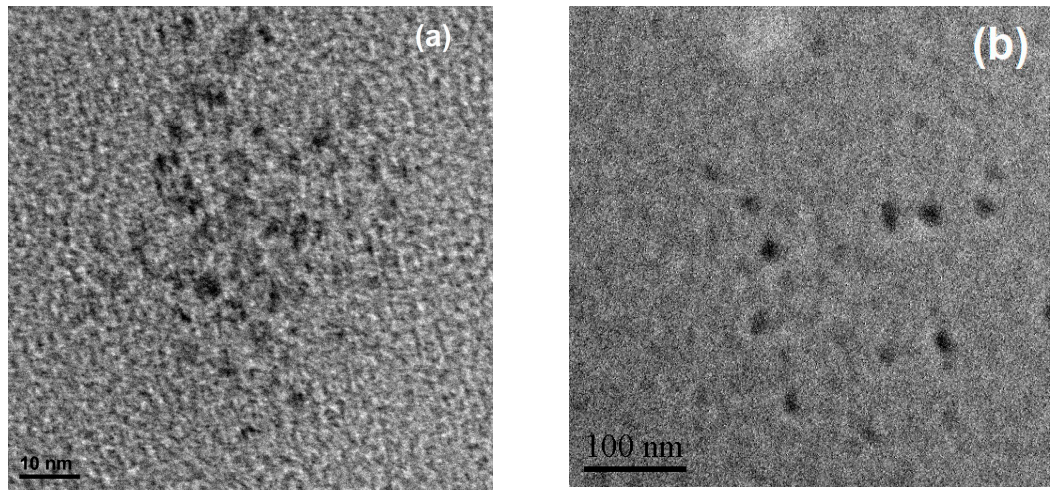

Figure 5. The TEM images of (a) b-SiQDs; (b) y-SiQDs. 


\subsubsection{FT-IR Spectrometer}

A FT-IR spectrometer was used to examine the functional groups on the surface of SiQDs. As shown in Figure 6, the surface functional groups of b-SiQDs, G-SiQDs, and y-SiQDs are quite similar, including the stretching vibration of $\mathrm{N}-\mathrm{H}$ [30], $\mathrm{O}-\mathrm{H}$ [31] at $3277 \mathrm{~cm}^{-1}$ and $3367 \mathrm{~cm}^{-1}$, and the stretching vibrations of $-\mathrm{CH}=\mathrm{N}-[32]$ at $1595 \mathrm{~cm}^{-1}$. Stretching and bending vibrations due to methyl groups [33] were represented by the bands at $2935 \mathrm{~cm}^{-1}$ and $1462 \mathrm{~cm}^{-1}$, the asymmetrical deformation vibration of $\mathrm{Si}-\mathrm{O}$ and the stretching vibrations of $\mathrm{Si}-\mathrm{O}-\mathrm{Si}$ at $1310 \mathrm{~cm}^{-1}$ and $1130-1010 \mathrm{~cm}^{-1}$ [27]. These results show that the existence of hydroxyl and ammonia groups could effectively enhance the water-solubility and stability of the prepared SiQDs. However, the spectra of b-SiQDs do not exhibit carbonyl groups [34] absorption peak at $1663 \mathrm{~cm}^{-1}$ as is the case with both $y$-SiQDs and g-SiQDs. The low vibration frequency of the carbonyl groups proves the presence of large conjugation systems on both sides of the carbonyl groups, leading to a weakened strength of the double bond. The enlarged conjugation system makes the $\lambda_{\mathrm{em}}$ redshift. The zeta potential of $\mathrm{y}$-SiQDs is $-17 \mathrm{mV}$, showing the surface of the SiQDs is negatively charged with the existence of hydroxyl group on the surface.

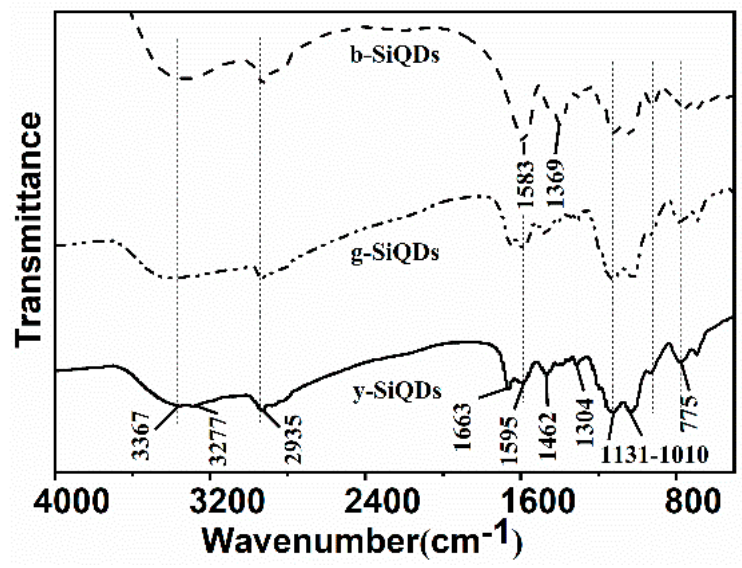

Figure 6. FT-IR spectrum of the three kinds of SiQDs (b-SiQDs, g-SiQDs, and y-SiQDs).

\subsubsection{EDS Spectrum}

The EDS spectrum was used to analyze the chemical constitutions of y-SiQDs and b-SiQDs (Figures S6 and S7). The contents of C, N, O, and Si elements in both SiQDs were measured, and their relative atomic weight ratios are listed in Figure 7. The relative atomic weight ratios of $\mathrm{C}$ to $\mathrm{O}$ of b-SiQDs and y-SiQDs are 1:0.05 and 1:0.168, respectively, showing a richer existence of $\mathrm{O}$ elements on the surface of $y$-SiQDs and thus a relative higher oxidation degree. This also proves the existence of carbonyl groups on the surface as shown from the FT-IR spectra of the y-SiQDs.

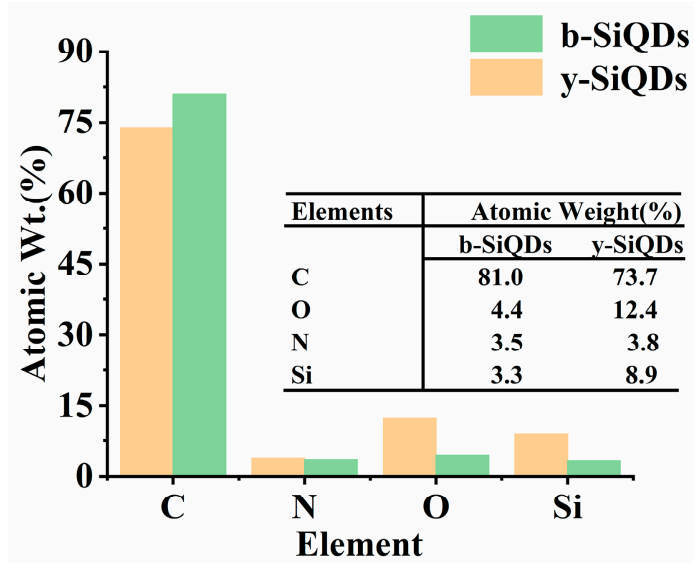

Figure 7. Atomic weight composition (\%) of C, N, O, and Si for b-SiQDs and y-SiQDs examined by EDS. 


\subsubsection{Study on the Synthesis Mechanism}

In our previous work [27], the QYs of SiQDs (with DAMO as the silicon source, sodium oxalate, and citric acid as the reducing agent pair) were effectively improved by synergistic effects, but the $\lambda_{\text {em }}$ did not change. In this paper, we selected the right pair of reducing agents (CC and Na-citrate), which not only improved the QYs of SiQDs, but also caused different degrees of red shift in the emission wavelengths.

Figure 8 exhibits the synthesis mechanism of b-SiQDs, g-SiQDs, and y-SiQDs. When we use DAMO as the silicon source and Na-citrate as the reducing agent, the silane DAMO undergoes hydrolysis-reduction-polymerization to form b-SiQDs [13]; when we use DAMO as the silicon source, Na-citrate and CC as the double reducing agent, y-SiQDs are synthesized. As a common excellent reducing agent, Na-citrate is beneficial for the rapid hydrolysis and reduction of silane to form a silicon core. In addition to participating in the reduction of silane during the synthesis, the phenolic hydroxyl group on the surface of the CC can be further combined with the residue on the surface of the hydrolyzed product of DAMO to form a fluorophore having a larger conjugated system and a $\mathrm{C}=\mathrm{O}$ structure on the surface, which is advantageous for the SiQDs to form long wavelengths and higher QYs. The presence of carbonyl groups on the surface of SiQDs was also confirmed by the higher O/C ratio of EDS observed in the IR spectra.

A
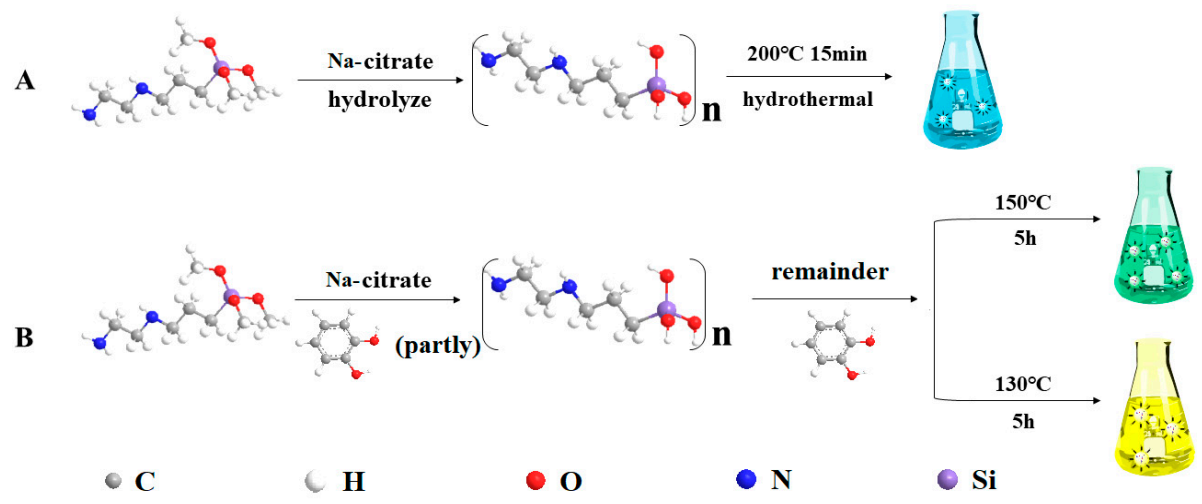

Figure 8. Schematic illustration of the synthesis of (A) b-SiQDs and (B) g-SiQDs and y-SiQDs.

It is worth noticing that lowering of either reaction temperature or time would make the emission wavelength of the prepared SiQDs redshift. This is different from the synthesis mechanism of other SiQDs. With the same reactants ratio and reaction time $(5 \mathrm{~h}), \mathrm{y}$-SiQDs can be acquired at a temperature of $130{ }^{\circ} \mathrm{C}$, while higher temperature $\left(150{ }^{\circ} \mathrm{C}\right)$ would lead to the formation of g-SiQDs. We suspect that the possible reason is that this is not conducive to the stable existence of the CC structure at high temperature conditions, and thus cannot participate in the formation of the surface luminescent groups of $y$-SiQDs.

\subsection{Application of the Prepared SiQDs}

\subsubsection{Optical Stability of the SiQDs}

The optical stability is one of the most crucial properties of QDs for their wide application in the fields of analytical detection, optical sensing, and nonlinear optical materials. The optical stability of prepared SiQDs was tested by setting it under natural light and measuring its fluorescence intensity every five minutes. The fluorescence intensities of both y-SiQDs and g-SiQDs exhibit no obvious decrease on $30 \mathrm{~min}$ of exposure to natural light (Figure S8), proving their excellent optical stability. 


\subsubsection{Cytotoxicity of the SiQDs}

The cytotoxicity of SiQDs is also an important factor with regard to their final application in an organism [35,36]. The MTT method is the common way to assess the cytotoxicity of QDs. y-SiQDs, and g-SiQDs in different concentrations were used to incubate the L02 cell for $24 \mathrm{~h}$, and then the activity of the cells was measured. Figure 9 shows that two kinds of SiQDs exhibit really low cytotoxicity with almost no interference to the growth of the cells. The excellent stability and safety could guarantee their potential application as probes in biochemical fields.

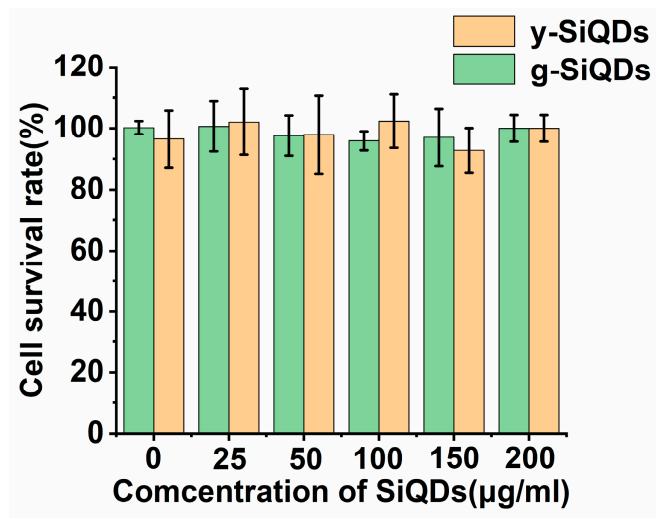

Figure 9. Cell viability (\%) measured by MTT assay. The L02 cells were incubated with the SiQDs for $24 \mathrm{~h}$ at $37^{\circ} \mathrm{C}$. All results were presented as the mean \pm standard deviation (SD) from three independent experiments with four wells in each.

\subsubsection{Fluorescence Ink}

Since y-SiQDs and g-SiQDs exhibit excellent optical stability and low toxicity at high concentration, they show great possibility as new biocompatible fluorescence inks. Figure 10a,b shows the pattern drawn with y-SiQDs and g-SiQDs on filter paper under natural light and UV light (365 nm). The filter paper exhibits a strong bright yellow and green fluorescence pattern under UV light. This proves that $\mathrm{y}$-SiQDs and g-SiQDs could be used as a fluorescence invisible ink, or further be applied in the field of banknote anti-counterfeiting techniques [37].

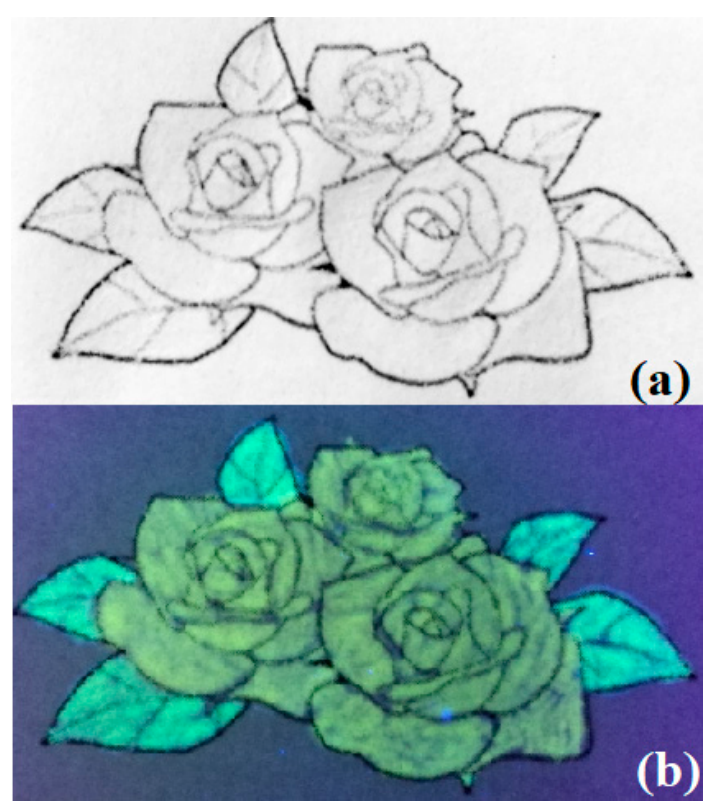

Figure 10. Photos of pictures painted by y-SiQDs and g-SiQDs. Photo (a) was photographed under daylight and (b) was photographed under UV-light (365 nm). 


\section{Conclusions}

This paper reports the preparation of stable y-SiQDs and G-SiQDs through the one-step hydrothermal route with synergistic effects between double reducing reagents CC and Na-citrate, with the silane coupling agent N-[3-(trimethoxysilyl)propyl]ethylenediamine (DAMO) as silicon source. The QYs of the prepared y-SiQDs and g-SiQDs reach as high as $29.4 \%$ and $38.3 \%$. The optical properties of both SiQDs were characterized and tested by three-dimensional fluorescence spectra, IR spectra, EDS, and TEM, with further comparison to the b-SiQDs prepared by DAMO and Na-citrate. It was discovered that the participation of the carbonyl group during the synthesis makes the $\lambda_{\mathrm{em}}$ redshift. The preparation of long-wavelength emitting SiQDs through a synergistic effect and their highly efficient and stable photoluminescence ensure their promising application as optical materials.

Supplementary Materials: The following are available online at http:/ www.mdpi.com/2079-4991/9/3/466/s1, Figure S1: UV-Vis adsorption and photoluminescence emission spectra of y-SiQDs; Figure S2: UV-Vis adsorption and photoluminescence emission spectra of g-SiQDs; Figure S3: Three-dimensional fluorescence spectra of three kinds of SiQDs(CC); Figure S4: (a) particle size distribution histograms and (b) lattice image y-SiQDs; Figure S5: (a) particle size distribution histograms and (b) lattice image b-SiQDs; Figure S6: EDS spectra of y-SiQDs; Figure S7: EDS spectra of b-SiQDs; Figure S8: The relationship of replaced time with the fluorescence intensity of y-SiQDs and g-SiQDs.

Author Contributions: Conceptualization, D.Z. and Z.Z.; methodology, D.Z. and Z.Z.; software, J.L.; project administration, D.Z., J.L., and X.X.; formal analysis, Z.Z.; investigation, Z.Z., C.W., and W.M.; resources, D.Z.; data curation, Z.Z.; writing-original draft preparation, Z.Z.; writing-review and editing, Z.Z. and D.Z.; visualization, Z.Z., C.W., and W.M.; supervision, D.Z.; funding acquisition, D.Z.

Funding: This research was funded by NATURAL SCIENCE FOUNDATION OF HUBEI PROVINCE, grant number 2016CFB615 and by FUNDAMENTAL RESEARCH FUNDS FOR THE "CENTRAL UNIVERSITIES", SOUTH CENTRAL UNIVERSITY FOR NATIONALITIES, grant number CZY19029, CZY15020, CZW15017 and XTZ15013.

Acknowledgments: The authors would like to thank Jianhua Wei for his technical support.

Conflicts of Interest: The authors declare no conflict of interest.

\section{References}

1. Shan, C.; Wu, K.; Yen, H.-J.; Narvaez Villarrubia, C.; Nakotte, T.; Bo, X.; Zhou, M.; Wu, G.; Wang, H.-L. Graphene Oxides Used as a New "Dual Role" Binder for Stabilizing Silicon Nanoparticles in Lithium-Ion Battery. ACS Appl. Mater. Interfaces 2018, 10, 15665-15672. [CrossRef] [PubMed]

2. Tang, W.; Guo, X.X.; Liu, X.H.; Chen, G.; Wang, H.J.; Zhang, N.; Wang, J.; Qiu, G.Z.; Ma, R.Z. Interconnected silicon nanoparticles originated from halloysite nanotubes through the magnesiothermic reduction: A high-performance anode material for lithium-ion batteries. Appl. Clay Sci. 2018, 162, 499-506. [CrossRef]

3. Tang, M.M.; Ji, X.Y.; Xu, H.; Zhang, L.; Jiang, A.R.; Song, B.; Su, Y.Y.; He, Y. Photostable and Biocompatible Fluorescent Silicon Nanoparticles-Based Theranostic Probes for Simultaneous Imaging and Treatment of Ocular Neovascularization. Anal. Chem. 2018, 90, 8188-8195. [CrossRef] [PubMed]

4. Geng, X.; Li, Z.H.; Hu, Y.L.; Liu, H.F.; Sun, Y.Q.; Meng, H.M.; Wang, Y.W.; Qu, L.B.; Lin, Y.H. One-Pot Green Synthesis of Ultrabright N-Doped Fluorescent Silicon Nanoparticles for Cellular Imaging by Using Ethylenediaminetetraacetic Acid Disodium Salt as an Effective Reductant. ACS Appl. Mater. Interfaces 2018, 10, 27979-27986. [CrossRef] [PubMed]

5. Wang, R.G.; Zhao, M.Y.; Deng, D.; Ye, X.; Zhang, F.; Chen, H.; Kong, J.L. An intelligent and biocompatible photosensitizer conjugated silicon quantum dots- $\mathrm{MnO}_{2}$ nanosystem for fluorescence imaging-guided efficient photodynamic therapy. J. Mater. Chem. B 2018, 6, 4592-4601. [CrossRef]

6. Liu, Z.H.; Li, Y.Z.; Li, W.; Xiao, C.; Liu, D.F.; Dong, C.; Zhang, M.; Makila, E.; Kemell, M.; Salonen, J.; et al. Multifunctional Nanohybrid Based on Porous Silicon Nanoparticles, Gold Nanoparticles, and Acetalated Dextran for Liver Regeneration and Acute Liver Failure Theranostics. Adv. Mater. 2018, 30, 1703393-1703403. [CrossRef] [PubMed]

7. Luo, L.; Song, Y.; Zhu, C.Z.; Fu, S.F.; Shi, Q.R.; Sun, Y.M.; Jia, B.Z.; Du, D.; Xu, Z.L.; Lin, Y.H. Fluorescent silicon nanoparticles-based ratiometric fluorescence immunoassay for sensitive detection of ethyl carbamate in red wine. Sens. Actuators B Chem. 2018, 255, 2742-2749. [CrossRef] 
8. Zhu, B.Y.; Ren, G.J.; Tang, M.Y.; Chai, F.; Qu, F.Y.; Wang, C.G.; Su, Z.M. Fluorescent silicon nanoparticles for sensing $\mathrm{Hg}^{2+}$ and $\mathrm{Ag}^{+}$as well visualization of latent fingerprints. Dyes Pigments 2018, 149, 686-695. [CrossRef]

9. Tu, C.C.; Hoo, J.H.; Bohringer, K.F.; Lin, L.Y.; Cao, G.Z. Red-emitting silicon quantum dot phosphors in warm white LEDs with excellent color rendering. Opt. Express 2014, 22, A276-A281. [CrossRef] [PubMed]

10. Kang, Z.; Liu, Y.; Tsang, C.H.A.; Ma, D.D.D.; Fan, X.; Wong, N.B.; Lee, S.T. Water-soluble silicon quantum dots with wavelength-tunable photoluminescence. Adv. Mater. 2009, 21, 661-664. [CrossRef]

11. Erogbogbo, F.; Yong, K.T.; Roy, I.; Xu, G.X.; Prasad, P.N.; Swihart, M.T. Biocompatible luminescent silicon quantum dots for imaging of cancer cells. ACS Nano 2008, 2, 873-878. [CrossRef] [PubMed]

12. Dohnalova, K.; Poddubny, A.N.; Prokofiev, A.A.; de Boer, W.D.A.M.; Umesh, C.P.; Paulusse, J.M.J.; Zuilhof, H.; Gregorkiewicz, T. Surface brightens up Si quantum dots: direct bandgap-like size-tunable emission. Light Sci. Appl. 2013, 2, 47-53. [CrossRef]

13. Xu, X.L.; Ma, S.Y.; Xiao, X.C.; Hu, Y.; Zhao, D. The preparation of high-quality water-soluble silicon quantum dots and their application in the detection of formaldehyde. RSC Adv. 2016, 6, 98899-98907. [CrossRef]

14. Ma, S.D.; Chen, Y.L.; Feng, J.; Liu, J.J.; Zuo, X.W.; Chen, X.G. One-Step Synthesis of Water-Dispersible and Biocompatible Silicon Nanoparticles for Selective Heparin Sensing and Cell Imaging. Anal. Chem. 2016, 88, 10474-10481. [CrossRef]

15. Wang, J.; Ye, D.X.; Liang, G.H.; Chang, J.; Kong, J.L.; Chen, J.Y. One-step synthesis of water-dispersible silicon nanoparticles and their use in fluorescence lifetime imaging of living cells. J. Mater. Chem. B 2014, 2, 4338-4345. [CrossRef]

16. Han, Y.X.; Chen, Y.L.; Feng, J.; Liu, J.J.; Ma, S.D.; Chen, X.G. One-Pot Synthesis of Fluorescent Silicon Nanoparticles for Sensitive and Selective Determination of 2,4,6-Trinitrophenol in Aqueous Solution. Anal. Chem. 2017, 89, 3001-3008. [CrossRef] [PubMed]

17. Srivastava, R.; Gupta, S.K.; Naaz, F.; Singh, A.; Singh, V.K.; Verma, R.; Singh, N.; Singh, R.K. Synthesis, antibacterial activity, synergistic effect, cytotoxicity, docking and molecular dynamics of benzimidazole analogues. Comput. Biol. Chem. 2018, 76, 1-16. [CrossRef] [PubMed]

18. Yang, Q.; Xu, Q.; Jiang, H.-L. Metal-organic frameworks meet metal nanoparticles: synergistic effect for enhanced catalysis. Chem. Soc. Rev. 2017, 46, 4774-4808. [CrossRef] [PubMed]

19. Liu, C.B.; Chu, X.J.; Sun, P.Y.; Feng, X.J.; Huang, W.W.; Liu, H.X.; Ma, Y.B. Synergy effects of Polyinosinic-polycytidylic acid, CpG oligodeoxynucleotide, and cationic peptides to adjuvant HPV E7 epitope vaccine through preventive and therapeutic immunization in a TC-1 grafted mouse model. Hum. Vaccines Immunother. 2018, 14, 931-940. [CrossRef]

20. Zhao, R.L.; He, Y.M. Network pharmacology analysis of the anti-cancer pharmacological mechanisms of Ganoderma lucidum extract with experimental support using Hepa1-6-bearing C57 BL/6 mice. J. Ethnopharmacol. 2018, 210, 287-295. [CrossRef]

21. Zhan, Y.Q.; Zhang, J.M.; Wan, X.Y.; Long, Z.H.; He, S.J.; He, Y. Epoxy composites coating with $\mathrm{Fe}_{3} \mathrm{O}_{4}$ decorated graphene oxide: Modified bio-inspired surface chemistry, synergistic effect and improved anti-corrosion performance. Appl. Surf. Sci. 2018, 436, 756-767. [CrossRef]

22. Zhang, L.; Zhang, G.; Li, Y.; Wang, S.; Lei, A. The synergistic effect of self-assembly and visible-light induced the oxidative $\mathrm{C}-\mathrm{H}$ acylation of $\mathrm{N}$-heterocyclic aromatic compounds with aldehydes. Chem. Commun. 2018, 54, 5744-5747. [CrossRef] [PubMed]

23. Chen, Y.Z.; Zhou, Y.X.; Wang, H.; Lu, J.; Uchida, T.; Xu, Q.; Yu, S.H.; Jiang, H.L. Multifunctional PdAg@ MIL-101 for one-pot cascade reactions: combination of host-guest cooperation and bimetallic synergy in catalysis. ACS Catal. 2015, 5, 2062-2069. [CrossRef]

24. Anastasaki, A.; Nikolaou, V.; Zhang, Q.; Burns, J.; Samanta, S.R.; Waldron, C.; Haddleton, A.J.; McHale, R.; Fox, D.; Percec, V.; et al. Copper(II)/Tertiary Amine Synergy in Photoinduced Living Radical Polymerization: Accelerated Synthesis of omega-Functional and alpha,omega-Heterofunctional Poly(acrylates). J. Am. Chem. Soc. 2014, 136, 1141-1149. [CrossRef]

25. Ruiz-Palomero, C.; Soriano, M.L.; Benitez-Martinez, S.; Valcarcel, M. Photoluminescent sensing hydrogel platform based on the combination of nanocellulose and $\mathrm{S}, \mathrm{N}$-codoped graphene quantum dots. Sens. Actuators B Chem. 2017, 245, 946-953. [CrossRef] 
26. Xiao, N.; Liu, S.G.; Mo, S.; Li, N.; Ju, Y.J.; Ling, Y.; Li, N.B.; Luo, H.Q. Highly selective detection of p-nitrophenol using fluorescence assay based on boron, nitrogen co-doped carbon dots. Talanta 2018, 184, 184-192. [CrossRef] [PubMed]

27. Ma, S.Y.; Yue, T.; Xiao, X.C.; Cheng, H.; Zhao, D. A proof of concept study of preparing ultra bright silicon quantum dots based on synergistic effect of reductants. J. Lumin. 2018, 201, 77-84. [CrossRef]

28. Wu, F.G.; Zhang, X.D.; Kai, S.Q.; Zhang, M.Y.; Wang, H.Y.; Myers, J.N.; Weng, Y.X.; Liu, P.D.; Gu, N.; Chen, Z. One-Step Synthesis of Superbright Water-Soluble Silicon Nanoparticles with Photoluminescence Quantum Yield Exceeding 80\%. Adv. Mater. Interfaces 2015, 2, 1500360-1500371. [CrossRef]

29. Ghosh, B.; Shirahata, N. Colloidal silicon quantum dots: synthesis and luminescence tuning from the near-UV to the near-IR range. Sci. Technol. Adv. Mater. 2014, 15, 014207-014221. [CrossRef]

30. Kumar, A.; Grewal, A.S.; Singh, V.; Narang, R.; Pandita, D.; Lather, V. Synthesis, Antimicrobial Activity and QSAR Studies of Some New Sparfloxacin Derivatives. Pharm. Chem. J. 2018, 52, 444-454. [CrossRef]

31. Selim, Y.; Abd El-Azim, M.H.M. Conventional and Microwave-Activated the Synthesis of a Novel Series of Imidazoles, Pyrimidines, and Thiazoles Candidates. J. Heterocycl. Chem. 2018, 55, 1403-1409. [CrossRef]

32. Oleynik, I.V.; Oleynik, I.I. Design of Postmetallocene Catalytic Systems of Arylimine Type for Olefin Polymerization: XVIII. Synthesis of N-Arylsalicylaldimine Ligands Containing meta-or para-Diallylamino Group and Their Titanium(IV) Complexes. Russ. J. Org. Chem. 2018, 54, 537-544. [CrossRef]

33. Musa, W.J.; Duengo, S.; Situmeang, B. Isolation and characterization triterpenoid compound from leaves mangrove plant (Sonnertia Alba) and antibacterial activity test. Int. Res. J. Pharm. 2018, 9, 85-89. [CrossRef]

34. Kyomugasho, C.; Christiaens, S.; Shpigelman, A.; Van Loey, A.M.; Hendrickx, M.E. FT-IR spectroscopy, a reliable method for routine analysis of the degree of methylesterification of pectin in different fruit- and vegetable-based matrices. Food Chem. 2015, 176, 82-90. [CrossRef] [PubMed]

35. Liu, P.; Behray, M.; Wang, Q.; Wang, W.; Zhou, Z.G.; Chao, Y.M.; Bao, Y.P. Anti-cancer activities of allyl isothiocyanate and its conjugated silicon quantum dots. Sci. Rep. 2018, 8, 1084. [CrossRef] [PubMed]

36. Cheng, X.Y.; McVey, B.F.P.; Robinson, A.B.; Longatte, G.; O’Mara, P.B.; Tan, V.T.G.; Thordarson, P.; Tilley, R.D.; Gaus, K.; Gooding, J.J. Protease sensing using nontoxic silicon quantum dots. J. Biomed. Opt. 2017, 22, 1-7. [CrossRef] [PubMed]

37. Song, B.; Wang, H.Y.; Zhong, Y.L.; Chu, B.B.; Su, Y.Y.; He, Y. Fluorescent and magnetic anti-counterfeiting realized by biocompatible multifunctional silicon nanoshuttle-based security ink. Nanoscale 2018, 10, 1617-1621. [CrossRef] [PubMed] 